\title{
Fingerprint Indexing Based on Composite Set of Reduced SIFT Features
}

\author{
Xin Shuai ${ }^{1}$, Chao Zhang $^{1}$ and Pengwei Hao ${ }^{1,2}$ \\ ${ }^{1}$ Key Laboratory of Machine Perception (Ministry of Education), \\ Peking University, Beijing 100871, China \\ ${ }^{2}$ Department of Computer Science, Queen Mary University of London, London E1 4NS, UK \\ chzhang@cis.pku.edu.cn
}

\begin{abstract}
Most of current fingerprint indexing schemes utilize features based on global textures and minutiae structures. To extend the existing technology of feature extraction, this paper proposes a new fingerprint indexing and retrieval scheme using scale invariant feature transformation (SIFT), which has been widely used in generic image retrieval. With slight loss in effectiveness, we reduce the number of features generated from one fingerprint for efficiency. To cope with the uncertainty of acquisition (e.g. partialness, distortion), we use a composite set of features to form multiple impressions for the fingerprint representation. In the index construction phase, the use of locality-sensitive hashing (LSH) allows us to perform similarity queries by only examining a small fraction of the database. Experiments on database FVC2000 and FVC2002 show the effectiveness of our proposed scheme.
\end{abstract}

\section{Introduction}

In a fingerprint identification system, once a user input a fingerprint, the system has to search a database to identify the corresponding one without a claimed identity. However, the size of very large databases seriously compromises the efficiency of the system [1]. This can be alleviated by the techniques of classification and indexing which reduce the search space. Classification techniques attempt to classify fingerprints into 5 exclusive classes. Due to the uneven natural distribution, comparatively large inter-class similarity and intra-class difference, the workload reduction resulted from classification is not gratifying [1][2].

Fingerprint indexing algorithms, which performs better than exclusive classification, select most probable candidates and sort them by the similarity to the input one [2][3][4][5]. Those methods can be divided into two categories: Global and Local based. For global based methods, the feature represents the global pattern of ridges with a uniform model. The algorithms using directional field and Gabor filtered image around the core point in [2] and [3] are of this category. However, such approaches are not particularly good at handling partialness or distortion, due to their wide and uniform model. For local based methods, features represents stable local structures which are believed robust to partial prints. In [4] and [5], the triplets of minutiae and accessorial information are used in the indexing procedure. Even if some features are missing, the object fingerprint can still be retrieved as long as enough local features are found and matched.

Due to the area limitation of solid-sensors and uncertainty of acquisition, partial fingerprints are usually obtained [6]. Templates based on such prints are not reliable and robust for database construction. According to the characteristics of fingerprints, local based methods are mostly based on minutiae points. However, since the quantity of minutiae points in partial prints is relatively small, the retrieval performance may be poor. In this work, we extend the existing technology of feature extraction by introducing reduced scale invariant feature transformation (SIFT) [7][10] as representation for fingerprint indexing. To cope with the drawback of using only one impression for indexing construction, we propose a method using composite local features. The use of locality-sensitive hashing (LSH) [8][9] ensures us an efficient performance.

The remainder of this paper is organized as follows. Section 2 describes the SIFT algorithm and introduces our SIFT reducing process. Section 3 details the composite set of reduced local features. Section 4 presents LSH scheme used for our index construction. Section 5 provides the implementation detail of the proposed algorithm. Section 6 shows the experimental results, and in Section 7 we end with some conclusions. 


\section{Reduced SIFT features for fingerprint lo- cal representation}

In the area of fingerprint indexing, we can distinguish between "Global" and "Local" approaches. There are two considerations to use a feature as a local descriptor for indexing. First, the feature should be localized and abstracted stably. Second, the neighborhood descriptor should be distinctive enough for accurate and efficient matching.

In the literature, local approaches mainly adopt minutiae for the interest points. Minutiae triplets are usually used as distinctive descriptors [4][5]. To extend the existing minutiae based techniques, we employ the commonly used scale invariant feature transformation (SIFT) [7] for local interest point detection and description.

\subsection{SIFT features in fingerprint images}

The SIFT descriptor, which is invariant to scale, rotation and affine transforms, has been demonstrated to be robust and efficient in object recognition and image retrieval [7][9]. There are four major stages in SIFT algorithm: (1) scale-space peak detection; (2) keypoint localization; (3) orientation assignment; (4) generation of local descriptor.

As features for fingerprint representation, SIFT features have two major advantages compared with minutiae. First, SIFT generates a large number of features over a broad range of scales and locations, while the number of minutiae points appearing in a plain fingerprint image impression is limited to a small number $(<100)$. Furthermore, the number of SIFT feature points can be regulated by a set of parameters such as the number of octaves and scales. Second, actually most of the minutiae points can also be detected by SIFT interest point detector. Figure 1 shows the comparison of quantities of minutiae and SIFT points in a fingerprint.

\subsection{Reduced SIFT features}

With the naive SIFT detector, the number of computed keypoints are typically on the order of $10^{3}$ (this depends on the area and complexity of the fingerprint image foreground). For retrieval tasks on large collections, such a large number of keypoints can reduce any efficient index structure to a sequential search [10]. For the task of fingerprint indexing and retrieval, the full set of detected keypoints are found redundant, and often a small subset will suffice. Furthermore, experiment shows that SIFT features of scale levels higher than 3.5

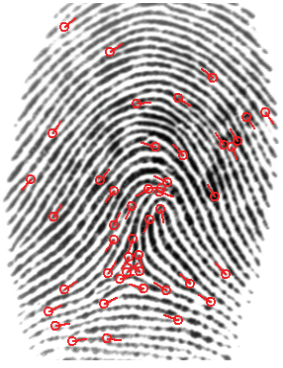

(a)

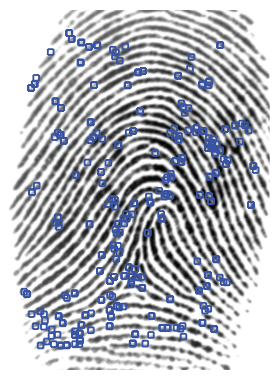

(c)

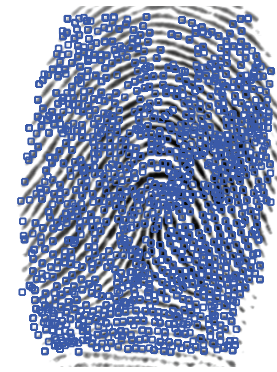

(b)

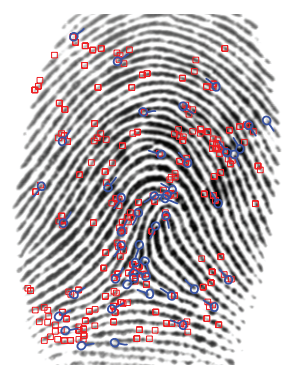

(d)
Figure 1. Minutiae and SIFT features on fingerprint. (a) 46 minutiae points,(b) 1952 SIFT points, (c) reduced SIFT points ( $N=$ 300), (d) minutiae (blue) and reduced SIFT (red) points on one print.

contribute little to the fingerprint matching. It is therefore desirable to reduce the quantity of keypoints generated to cut down the retrieval time.

We propose two steps to reduce the SIFT points. First, we limit the scale factor under a threshold (3 in our experiment); second, we vary the threshold applied to discard candidate local peaks, specifically the low contrast (intensity) threshold. To obtain a stable number of keypoints from each fingerprint, we simply select top $N$ most significant keypoints ranked by the contrast value. Figure 1 presents both the minutiae and reduced SIFT points on a fingerprint. We can see that there are many SIFT features around each minutiae points.

The reducing strategy enables fingerprints to be indexed using only a small subset of keypoints with slight loss in effectiveness and great improvement in efficiency during retrieval.

\section{Composite set of local descriptors}

Registering fingerprint images is a difficult problem for the following reasons: (1) multiple impressions of the same finger acquired by fingerprint sensors may have a small region of overlap; (2) impressions may have different non-linear plastic distortions due to the 
effect of pressing a convex elastic surface (the finger) on a solid flat surface (the sensor). Moreover, these distortions may be present only in certain regions of the sensed image due to the non-uniform pressure applied by subject. (3) The presence of dirt deposits on the finger or the sensor can result in a rather noisy image.

To deal with these problems, different schemes have been developed that construct a composite template using multiple impressions [6]. For global representation approaches, the impressions must be stitched together to generate a composite image. In approaches with local descriptors, a composite set of local features that representing the all-around fingerprint and the possible distortions that may occur should be built.

In the proposed algorithm, we build the composite set of local features by integrating the reduced SIFT features extracted from three randomly selected impressions of the same finger.

\section{LSH for index construction}

To index the set of 128-dimensional SIFT descriptors, we use the locality-sensitive hashing (LSH) [8][9] algorithm. LSH is an approximate similarity search technique that works efficiently even for highdimensional data. It has been shown that LSH outperforms tree-based structures such as the SR-tree by at least an order of magnitude [8].

LSH solves the following approximate nearest neighbor search problem, termed $\epsilon$-NNS, in sub-linear time. Given a set of points $\mathcal{P}$ in $d$-dimensional space, we want to preprocess $\mathcal{P}$ such that given any point query $q$, we can quickly find an $(1+\epsilon)$-approximate nearest neighbor of $q$ in $\mathcal{P}$. We call $p^{*}$ an $(1+\epsilon)$-approximate nearest neighbor of $q$ if for any $p \in \mathcal{P}$,

$$
d\left(p^{*}, q\right) \leq(1+\epsilon) d(p, q) .
$$

where $d(x, y)$ is the distance between $x$ and $y$.

This is accomplished using a set of locality sensitive hash functions. Given a set of points $\mathcal{P}$ and a similarity function $\operatorname{sim}(x, y)$, a locality sensitive hash function family $\mathcal{F}$ operates on $\mathcal{P}$, such that for any $x, y \in \mathcal{P}$,

$$
\operatorname{Prob}_{h \in \mathcal{F}}[h(x)=h(y)]=\operatorname{sim}(x, y) .
$$

By using such a function family, LSH reduces the rate of false negatives.

We use the popular algorithm for LSH in our scheme, introduced by Gionis in [8]. The algorithm conceptually transforms the points space $\mathcal{P}$ into a Hamming space where $L 1$ distance between points in the original space are preserved. $l$ hash functions that simply select $k$ bits of points in the transformed space are created. These $k$ bits are hashed to index into the buckets in the hash table. The two parameters, $k$ and $l$ enable us to select an appropriate trade-off between accuracy and running time. In our experiments, we use $k=200$ and $l=20$.

\section{Implementation of the algorithm}

This section describes the implementation details of our algorithm. There are mainly two phases. First, the index construction phase builds the composite set of reduced SIFT descriptors of each fingerprint image in the database, and indexes all of the local features via LSH. Then, in the query phase, the user can perform queries to find the corresponding fingerprint.

Indexing. First, we randomly select 3 fingerprint images acquired from the same finger. A fingerprint enhancement algorithm is performed on each image to avoid the affection of noise. Then, the reduced SIFT features $(N=200$ in our experiment) are extracted. Note that the keypoints on the boundary of the fingerprint foreground are almost unreliable, thus those keypoints are removed during the feature extraction phase. Finally, we create the LSH indexing structure using the composite set of reduced SIFT features of the three impressions.

Query. When the user queries a fingerprint image, we perform the enhancement, the extraction of reduced SIFT features as described above. Then, for each descriptor, we calculate the bucket ids using the $l$ locality hash functions and find out all the keypoints within those buckets to form the candidate list. All these candidate keypoints are checked by $L 2$ norm to find the nearest keypoint of each query keypoint. Finally, the retrieved nearest keypoints vote for the finger they belong to. The vote result shows the similarity between the query finger and the ones in the database.

\section{Experimental results}

The performance of the proposed algorithm has been evaluated on FVC2000 DB2 and FVC2002 DB1, on which many published results are based. Both the databases contain images of 100 different fingers with 8 impressions of 500-dpi resolution for each finger. The sizes of images in the two databases are $364 \times 256$ and $388 \times 374$, respectively.

Accuracy and efficiency are two main indications of the retrieval performance. In the experiments, the retrieval accuracy is calculated by the percentage of the input fingerprints whose corresponding ones in the data base to be correctly retrieved. The retrieval efficiency is indicated by a so-called penetration rate, which is the 


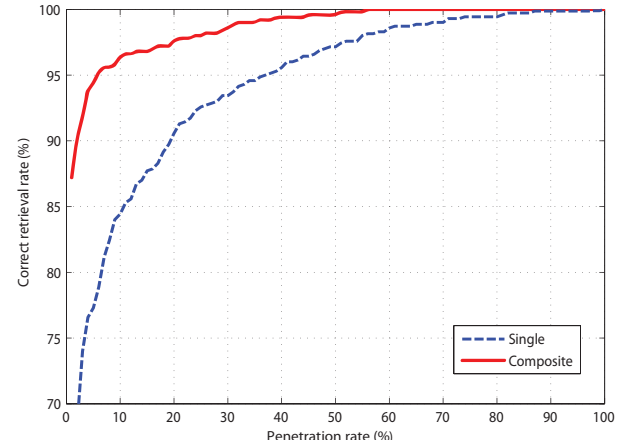

Figure 2. Comparison of performances by "Single" and "Composite" ways on FVC2002 DB1.

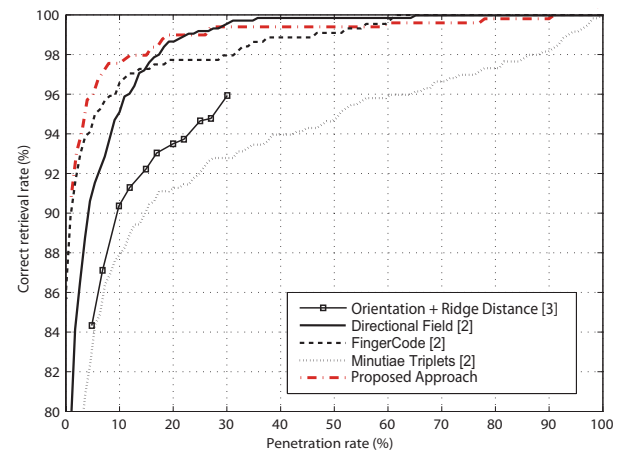

Figure 3. Retrieval results of the proposed approach and the approaches in [2] and [3] on FVC2000 DB2.

average percentage of fingerprints in the data base retrieved over all input fingerprints.

We have conduct experiments by ways of "Single" (using keypoints from only one impression for index construction) and "Composite" (using Composite set of keypoints from three impressions for index construction ). In the "Single" way, we randomly select one of the 8 impressions of the same finger to construct the database, while the other 7 prints serve as the test input fingerprint. In the "Composite" way, 3 impressions are randomly selected from the 8 impressions for the composite local feature based construction, and the other 5 are used for testing. Figure 2 shows the experimental results via these two approaches on FVC2002 DB1. The "Composite" approach performs much better than the "Single" one.

In the other experiment we perform only the "Composite" way on the database FVC2000 DB2. The results is shown in Figure 3. We also present some results on the same database: the methods based on directional field, FingerCode and minutiae triplets reported in [2], and the method combining orientation field and dominant ridge distance reported in [3].

\section{Conclusions}

This paper proposes a fingerprint indexing scheme based on composite set of reduced SIFT local features. The primary contributions of this paper are: (1) we introduce SIFT features for fingerprint indexing, which have advantage over those minutiae based features. (2) we present a reducing procedure for SIFT, which results in slight loss in effectiveness but great improvement in efficiency during retrieval. (3) we use multiple impressions to create a composite set of local features for indexing, which is demonstrated more efficient. Moreover, LSH is adopted in our high dimensional index construction.

ACKNOWLEDGEMENT This work is supported by research funds of NSFC No.60572043 and the NKBRPC No.2004CB318005.

\section{References}

[1] D. Maltoni, D. Maio, A. K. Jain, and A. Prabhakar. Handbook of fingerprint recognition. New York: Springer, 2003.

[2] J.D. Boer, A.M. Bazen and S.H. Gerez. Indexing fingerprint database based on multiple features. Proc. ProRISC, 12th Annual Workshop on Circuits, Systems and Signal Processing, 2001.

[3] X.D. Jiang, M. Liu and A. Kot. Fingerprint retrieval for identification. IEEE Trans. Information Forensics and Security, 1(4): 532-542, 2006.

[4] R.S. Germain, A. Califano, and S. Colville. Fingerprint matching using transformation parameter clustering. IEEE Comput. Sci. Eng. Mag., 4(4): 42-49, 1997.

[5] B. Bhanu and X. Tan. Fingerprint indexing based on noevel features of minutiae triplets. IEEE Trans. Pattern Anal. and Mach. Intell., 25(5): 616-622, 2003.

[6] A.K. Jain and A. Ross. Fingerprint mosaicking. Proc. Int. Conf. on Acoustics, Speech, and Signal Processing (ICASSP), 2002.

[7] D. G. Lowe. Distinctive image features from scaleinvariant keypoints. Int. Journal of Computer Vision, 60(2): 91-110, 2004.

[8] A. Gionis, P. Indyk, and R. Motwani. Similarity search in high dimensions via hashing. Proc. 25th VLDB Conf., 518-529, 1999.

[9] Y. Ke, R. Sukthankar and L. Huston. Efficient nearduplicate detection and sub-image retrieval. Proc. ACM Multimedia Conf., 869-876, 2004.

[10] J. J. Foo and R. Sinha. Pruning SIFT for scalable near-duplicate image matching. Proc. 18th Australasian Database Conf. (ADC2007), 63-71, 2007. 